\title{
Bacterial microbiota compositions of naturally fermented milk are shaped by both geographic origin and sample type
}

\author{
Z. Zhong, Q. Hou, L. Kwok, Z. Yu, Y. Zheng, Z. Sun, B. Menghe, and H. Zhang ${ }^{1}$ \\ Key Laboratory of Dairy Biotechnology and Engineering, Ministry of Education, Department of Food Science and Engineering, \\ Inner Mongolia Agricultural University, Hohhot, Inner Mongolia, 010018, P. R. China
}

\begin{abstract}
Naturally fermented dairy products contain a rich microbial biodiversity. This study aimed to provide an overview on the bacterial microbiota biodiversity of 85 samples, previously collected across a wide region of China, Mongolia, and Russia. Data from these 85 samples, including 55 yogurts, 18 naturally fermented yak milks, 6 koumisses, and 6 cheeses, were retrieved and collectively analyzed. The most prevalent phyla shared across samples were Firmicutes, Proteobacteria, Bacteroidetes, and Actinobacteria, which together accounted for $99 \%$ of bacterial sequences. The predominant genera were Lactobacillus, Lactococcus, Streptococcus, Acetobacter, Acinetobacter, Leuconostoc, and Macrococcus, which together corresponded to $96.63 \%$ of bacterial sequences. Further multivariate statistical analyses revealed significant differences in the microbiota structure across sample geographic origin and type. First, on the principal coordinate score plot, samples representing the 3 main sample collection regions (Russia, Xinjiang, and Tibet) were mostly located respectively in the upper left, lower right, and lower left quadrants, although slight overlapping occurred. In contrast, samples from the minor sampling areas (Inner Mongolia, Mongolia, Gansu, and Sichuan) were predominantly distributed in the lower left quadrant. These results suggest a possible association between sample geographical origin and microbiota composition. Second, bacterial microbiota structure was stratified by sample type. In particular, the microbiota of cheese was largely distinct from the other sample types due to its high abundances of Lactococcus and Streptococcus. The fermented yak milk microbiota was most like that of the yogurts. Koumiss samples had the lowest microbial diversity and richness. In conclusion, both geographic origin and sample type shape the microbial diversity of naturally fermented milk.
\end{abstract}

Received December 27, 2015.

Accepted April 2, 2016.

${ }^{1}$ Corresponding author: hepingdd@vip.sina.com
Key words: fermented milk, microbial diversity, pyrosequencing, bacterial composition

\section{INTRODUCTION}

The history of naturally fermented milk goes back thousands of years. At first, naturally fermented milk was manufactured in an artisanal and traditional way to prolong the shelf life of milk (Zamfir et al., 2006). More recently, it has become a very popular food in the human daily diet and fermented milk products play an important nutritional role in modern life. Until now, these traditional dairy products have been a vital component in the daily diet of herdsmen all around the world, as well as some ethnic groups such as the Mongolians. In addition to the intrinsic nutritional content of these dairy products, naturally fermented milks may offer extra health benefits and better palatability to consumers compared with the industrially manufactured milks due to the presence of a rich and highly diverse microbial population. Microbial diversity greatly contributes to the taste, flavor, texture, color, and nutritional properties of the resultant fermented dairy products (Marshall and Tamime, 1997; Oberman and Libudzisz, 1998).

The earliest studies on the microbial diversity of naturally fermented milk date from the end of nineteenth century (Oberman and Libudzisz, 1998), and the usual way of detecting the composition of the microbiota has been through culture methods (Mayo et al., 2014). These traditional methods mainly rely on the isolation and cultivation of microorganisms, followed by their identification and typing. However, the approach is often limited by the difficulty in simulating the natural habitat growth conditions that are essential for growing fastidious microbes. Indeed, most environmental microbes are known to be uncultivable, so a culturedependent technique always underestimates microbial diversity and sometimes fails to detect certain microbial groups (Oberman and Libudzisz, 1998; Ercolini et al., 2001). In recent decades, several culture-independent, DNA-based techniques, such as denaturing gradient gel electrophoresis, single-strand conformational poly- 
morphism, and DNA microarray methods, which can overcome the limitation of culture-dependent methods, have been widely used in evaluating the microbial composition and diversity of fermented foods (Giraffa and Neviani, 2001; Jany and Barbier, 2008). Among these methods, parallel high-throughput pyrosequencing is more sensitive. As a next-generation DNA sequencing platform, it can produce exceedingly high numbers of nucleic acid sequences and allow an in-depth characterization of the microbial constituents of ecosystems (Mayo et al., 2014). This technique has been widely adopted in evaluating microbial communities in various fermented foods, such as kefir (Dobson et al., 2011; Leite et al., 2012), cheese (Masoud et al., 2011; Alegría et al., 2012), pearl millet slurries (Humblot and Guyot, 2009), sourdoughs (Lattanzi et al., 2013), and fermented soybeans (Nam et al., 2012).

Our laboratory has previously performed extensive studies in characterizing the microbial diversity and communities in various types of naturally fermented dairy products collected over a wide geographical region across China, Mongolia, and Russia. In particular, we applied the parallel high-throughput pyrosequencing method to analyze the microbiota composition in naturally fermented cow (yogurt) and yak milk of China and Mongolia (Sun et al., 2014), yogurt in Xinjiang of China (Xu et al., 2015) and Russia (Liu et al., 2015a), fermented yak milk in Tibet of China (Liu et al., 2015b), koumiss (naturally fermented mare milk) and cheese of Xinjiang of China and Russia [J. Li, Y. Zheng, H. Xu, X. Xi, Q. Hou, S. Feng, W. Laga, L.-y. Kwok, Z. Sun, and T. Sun (Inner Mongolia Agricultural University, Hohhot, China), unpublished data]. To provide an overview of the microbial diversity of naturally fermented dairy products and evaluate the factors that drive such a rich biodiversity, we pooled and reanalyzed all data from 85 previously collected fermented dairy samples originating from different milk sources and geographical locations.

\section{MATERIALS AND METHODS}

\section{Samples and Pyrosequencing Data}

Eighty-five naturally fermented milk samples were reanalyzed in this study, including 55 yogurts, 18 yak milks, 6 koumisses, and 6 cheeses. The geographical origins of samples are listed in Supplemental Table S1 (http://dx.doi.org/10.3168/jds.2015-10825). The pyrosequencing data of the samples were retrieved from the MG-RAST database (http://metagenomics.anl.gov/; Accession numbers: 2912, 4509270-4509296 (Sun et al., 2014); 4570725.3-4570765.3 and 4576499.3 (Xu et al., 2015); 4570605.3-4570640.3 (Liu et al., 2015a); and 4572382.3-4572412.3 (Liu et al., 2015b).

\section{Sequence Processing and Bioinformatics Analysis}

In this study, the V3 region of the $16 \mathrm{~S}$ rRNA gene of 16 samples from Mongolia, Inner Mongolia, Gansu, and Sichuan and the V1-V3 region of the other 68 samples were amplified. Low-quality sequences were removed based on 2 sets of criteria. For the 16 samples from Mongolia, Inner Mongolia, Gansu, and Sichuan, sequences were removed if (1) raw reads were shorter than 140 nucleotides, (2) sequences had imperfect matches to the barcode, (3) sequences displayed a fuzzy match to at least one end of the primers, or (4) sequences had more than one ambiguous base. For the other 68 samples, sequences were removed if (1) raw reads were shorter than 300 nucleotides, (2) sequences had imperfect matches to the barcode, (3) sequences displayed a fuzzy match to at least one end of the primers, (4) sequences had only a short variable region of less than 300 nucleotides, or (5) raw reads had a quality score of $<20$ in more than $7 \%$ of the bases in the variable region.

Bioinformatics analyses were performed using the QIIME package (v1.2.1; Caporaso et al., 2010). First, the reads of $16 \mathrm{~S}$ rRNA variable regions were directly searched by BLAST (https://blast.ncbi.nlm.nih.gov/ Blast.cgi) against the Greengenes Database (http:// greengenes.lbl.gov; DeSantis et al., 2006). These retrieved 16S rRNA gene sequences were then used as representative sequences to identify operational taxonomic units (OTU) under the threshold of $97 \%$ identity using UCLUST method (Edgar, 2010). The potential chimeric sequences in the representative set of OTU were removed by ChimeraSlayer (Haas et al., 2011). The Shannon-Wiener, Simpson's diversity, Chao1, and rarefaction estimators were calculated to evaluate the $\alpha$ diversity. UniFrac (Lozupone and Knight, 2005) metrics were calculated to evaluate the $\beta$ diversity. Both weighted and unweighted calculations were performed for the principal coordinate analysis ( PCoA) and the multivariate ANOVA (MANOVA).

\section{Statistical Analyses}

Differences in the microbial populations between sample groups were assessed using the Kruskal-Wallis test and MANOVA. The permutational ANOVA (PERMANOVA) test was performed to detect the effect of geographical origin and milk animal source on the biodiversity of naturally fermented milk. 


\section{RESULTS}

\section{Richness and Diversity Analysis}

After preprocessing, a total of 860,731 high-quality reads were generated from the 85 naturally fermented milk samples, with an average of 10,126 reads for each sample (range $=1,390-22,097 ; \mathrm{SD}=19,837$ ). After BLAST analysis of the high-quality reads against the Greengenes Database (DeSantis et al., 2006), 1,602 bacterial OTU were identified at a $97 \%$ similarity level, with an average of 64.6 OTU per sample (range = 19-299; SD = 49.45). The Shannon diversity, but not rarefaction curves, reached the saturation phase for all samples (Figure 1). These results indicated that the majority of bacterial phylotypes present in the samples had already been captured in current sequencing depth, although new phylotypes would be expected by further sequencing.

Both the Shannon and Simpson's diversity indices were significantly different across the 4 kinds of naturally fermented milks (Table 1). Yak milk samples ranked highest based on these 2 diversity indices, followed by cheese and yogurt, whereas koumiss had the lowest diversity. Meanwhile, microbial richness was measured by the Chao1 index and the number of observed species (Table 1). Cheese samples showed the greatest microbial richness, followed by yogurt, yak milk, and koumiss. Our results suggest that microbial diversity and richness might not be directly correlated.

\section{Bacterial Composition of Naturally Fermented Milks}

Figure 2A shows the relative abundances of bacteria in 85 naturally fermented milk samples at the phylum level. The predominant phyla were Firmicutes, Proteobacteria, Bacteroidetes, and Actinobacteria, which together accounted for 99\% (99.59-100\%, SD = 0.05\%) of the bacterial sequences. Minor phyla included Cyanobacteria, Thermi, Acidobacteria, Tenericutes, TM7, and Verrucomicrobia.

At the genus level, 135 genera were detected in the 85 samples. The predominant genera were Lactobacillus, Lactococcus, Streptococcus, Acetobacter, Acinetobacter, Leuconostoc, and Macrococcus (Figure 2B), which together accounted for 96.63\% (37.57-100\%, $\mathrm{SD}=9.47 \%$ ) of the bacterial sequences. Furthermore, bacterial compositions were largely different across the 4 types of naturally fermented milk at the genus level with predominant genera of Lactococcus (55.42\%) and Streptococcus (31.90\%) in cheese, Lactobacillus $(82.75 \%)$ and Streptococcus $(13.93 \%)$ in koumiss, Lactobacillus (78.44\%), Streptococcus (14.08\%), and Lactococcus $(5.30 \%)$ in fermented yak milk, and Lacto- bacillus $(64.69 \%)$, Lactococcus $(14.62 \%)$, Streptococcus $(10.29 \%)$, and Acetobacter (4.78\%) in yogurt (Table 2).

Furthermore, we were able to identify specific correlations between some genera (Figure 3). The relative abundance of Lactobacillus was negatively correlated with Streptococcus, Lactococcus, Enterococcus, Acinetobacter, Leuconostoc, Acetobacter, and Macrococcus, whereas Lactococcus, Enterococcus, Acinetobacter, Leuconostoc, and Macrococcus were positively correlated. Thus, inter-genus competition or antagonism might have existed within the milk matrix environment.

\section{Multivariate Statistical Analyses of Microbiota Structure of Naturally Fermented Milk}

Multivariate statistical analyses were performed to compare the overall structure of microbial communities of all samples. Principal coordinate analysis based on the unweighted UniFrac distances revealed distinct microbiota structures across the koumiss, fermented yak milk, and cheese samples, whereas the yogurt samples showed no clustering pattern on the score plot (principal coordinates represented 12.42 and $8.90 \%$ of the total variance, respectively; Figure 4B). These results may indicate that microbial communities of yogurt samples are more complex than those of the other sample types.

The geographical origins of the samples are denoted by open or filled symbols of different shapes in Figure 4B. Symbols representing the 3 main sample collection regions (Russia, Xinjiang, and Tibet) were mostly located respectively in the upper left, lower right, and lower left quadrants of the score plot, although slight overlapping occurred. In contrast, symbols representing the minor sampling areas (Inner Mongolia, Mongolia, Gansu, and Sichuan) were predominantly distributed in the lower left quadrant. These results together suggest a possible association between sample geographical origin and microbiota composition of naturally fermented dairy products.

To further confirm whether the 2 factors, sample geographical origin and type, influence the naturally fermented milk microbiota biodiversity, 2 PERMANOVA tests were performed. First, to test the effect of geographical origin, we analyzed the 55 yogurt samples, which were all produced with cow milk but originated from several regions: Russia (19), Mongolia (3), Gansu (3), Sichuan (3), Inner Mongolia (5), and Xinjiang (22). The sample microbial biodiversity was significantly different across the samples of various geographical origins $(F=6.066, P=0.001)$. Second, to test the effect of sample type, the 28 Russian samples, including 19 yogurts, 3 koumisses, and 6 cheeses, were included in the PERMANOVA test calculation. No significant 

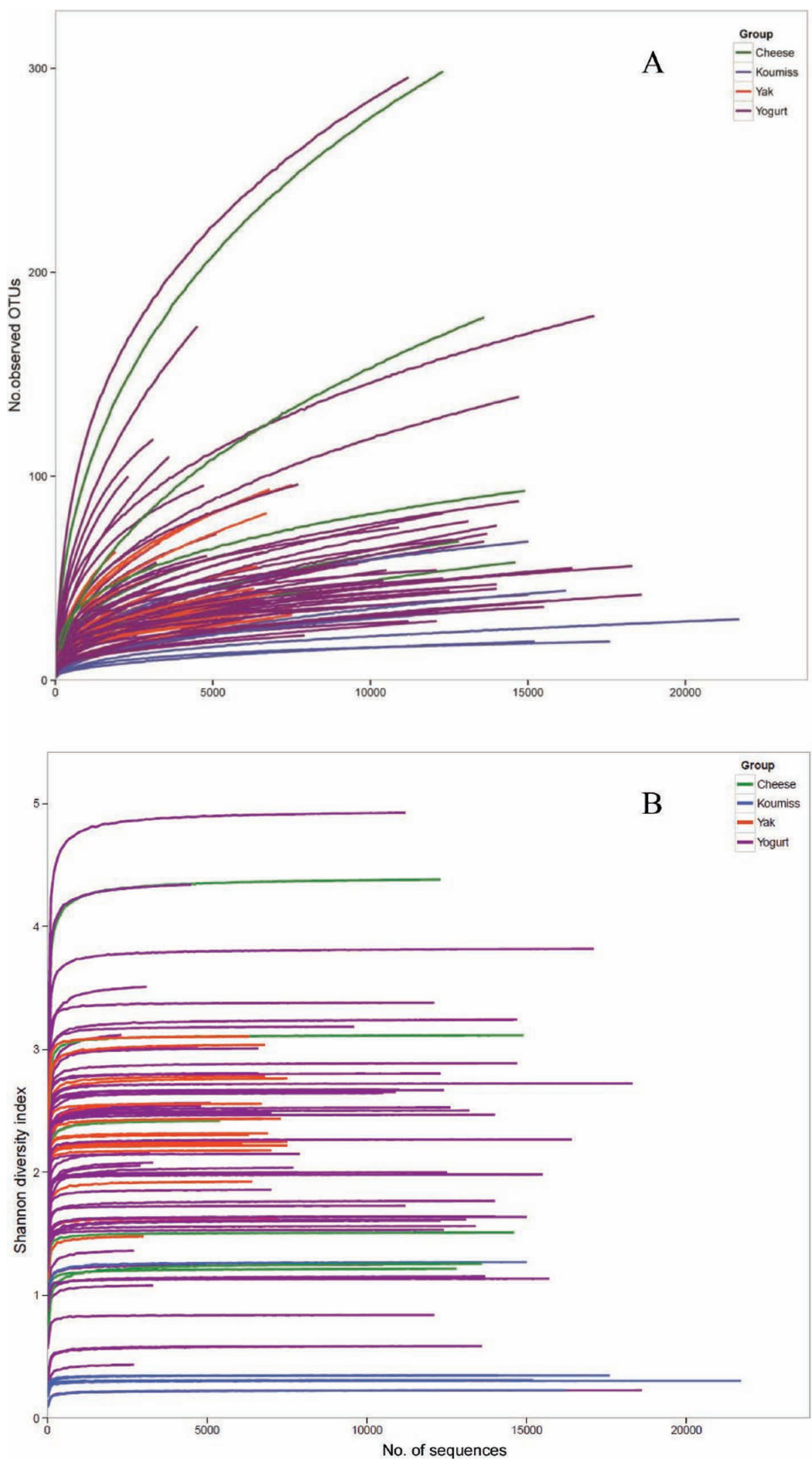

Figure 1. (A) Shannon diversity curves of bacterial populations of samples, and (B) rarefaction (operational taxonomic units, OTUs). Color version available online. 
Table 1. Sequence abundance and microbial diversity in the naturally fermented milk

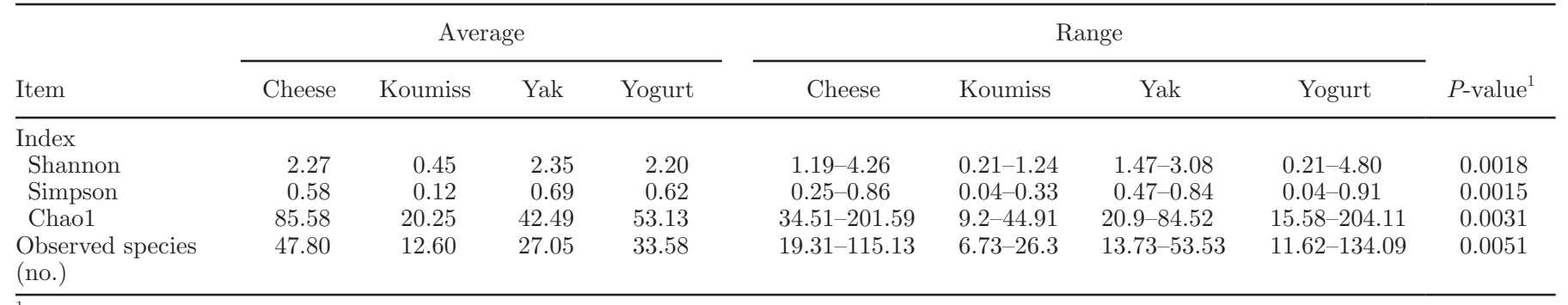

${ }^{1}$ Kruskal-Wallis test was used.

sample biodiversity difference was found among the 3 sample types $(F=1.510, P=0.119)$.

A MANOVA based on weighted (Figure 5A) and unweighted (Figure 5B) UniFrac distances was performed to compare the microbiota of different types of naturally fermented milk. The dendrograms built based on both weighted and unweighted UniFrac distances showed that cheese and koumiss samples each had a distinct microbiota composition, as demonstrated by the low $P$-values generated by the intergroup comparison. The microbiota composition of fermented yak milk and yogurt shared greater similarity, even though a significant difference was detected by the unweighted $(P<0.05)$ but not weighted UniFrac MANOVA.

\section{DISCUSSION}

This study aimed to provide a broad overview on the microbiota diversity of naturally fermented dairy products across a wide region of China, Mongolia, and Russia. Thus, we reanalyzed the $16 \mathrm{~S}$ rRNA gene pyrosequencing data of 85 naturally fermented milk samples. For 16 of the samples (from Mongolia, Inner Mongolia, Gansu and Sichuan), the V3 region was amplified, and for the other 68 samples, we targeted the V1-V3 region. One apparent problem was the difficulty in identifying unique representative sequences by a straightforward DNA alignment approach. To overcome this concern, we improved our operational taxonomic unit (OUT) identification procedure by directly blasting the obtained $16 \mathrm{~S}$ rRNA variable sequences against the Greengenes Database (http://greengenes.lbl.gov). Such a method makes it possible to compare the sequence reads amplified from different variable regions under a unified standard.

After the reanalysis, we identified 135 genera across the 85 samples. However, these genera were distributed unevenly, with only several core genera-Lactobacillus, Streptococcus, and Lactococcus - predominating. Lactobacillus was the most dominant genus in koumiss, fermented yak milk, and yogurt but not in cheese. Lactobacillus are basic lactic acid bacteria in milk fermen- tation (Huys et al., 2006) that degrade the disaccharide lactose, the major carbohydrate of milk. In addition to carbohydrate metabolism, other metabolic properties of the Lactobacillus, such as proteolytic and lipolytic capacities, contribute significantly to the texture, taste, and aroma of fermented milk (Holzapfel and Wood, 2014). Streptococcus is another important genus that exists stably in naturally fermented milk (ranging from 10.29 to $31.90 \%)$. Streptococcus thermophilus is one of the most commonly used starter cultures in milk fermentation (Hols et al., 2005). Apart from its role in contributing to product texture, taste, and aroma, Strep. thermophilus generally produces bacteriocins that protect the final product from microbial spoilage (Kabuki et al., 2009). Lactococcus accounted for a high proportion in the investigated fermented milks; in particular, this genus accounted for $55.42 \%$ in cheese. These results are in line with some previous studies (Alegría et al., 2012; Quigley et al., 2012). The major functions of these 3 genera of lactic acid bacteria are the production of lactic acid, hydrolysis of casein, lipolysis of fat, and fermentation of citric acid (Holzapfel and Wood, 2014). Their metabolic end-products and enzymes have a significant influence on the texture and flavor of naturally fermented milk; thus, Lactobacillus, Streptococcus, and Lactococcus could be defined as the core microbiota in naturally fermented milk.

One interesting observation found in our study was the large distinction of the cheese microbiome from the other sample types, characterized by the existence of a much higher proportion of Lactococcus and Streptococcus. We speculate that some specific steps during the cheese manufacturing process resulted in this unique bacterial makeup. For example, the process of salting before cheese ripening may suppress most lactic acid bacteria while enriching the halophilic Lactococcus (Sakala et al., 2002; Holzapfel and Wood, 2014). A harsher heating step $\left(35-55^{\circ} \mathrm{C}\right)$ that forces more whey from the curd is used to produce hard cheeses, which simultaneously selects for thermophilic bacteria such as Streptococcus. These processing conditions may explain the almost 3-fold higher abundance (31.9\%) of Strepto- 

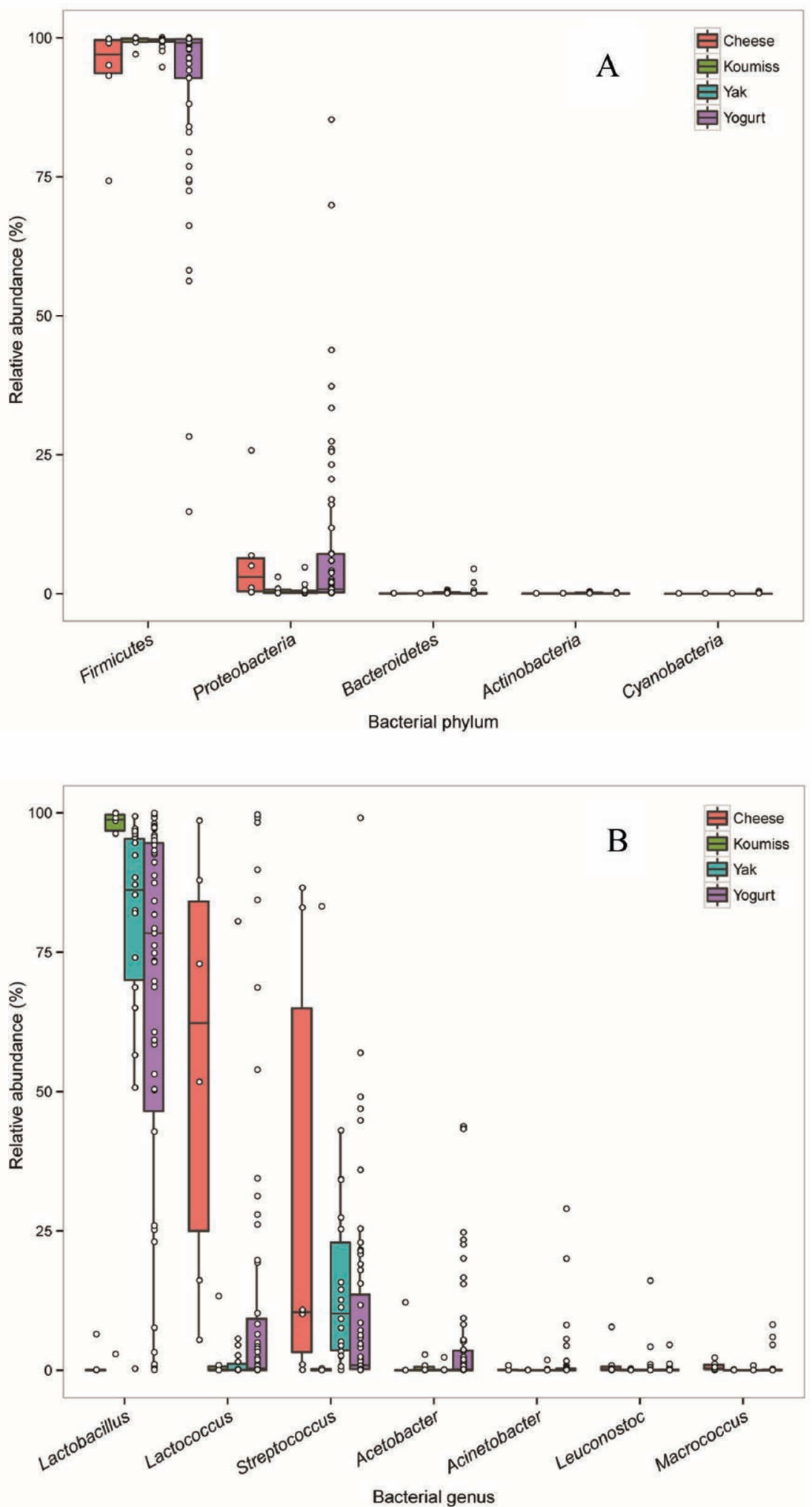

Figure 2. Relative abundance of bacterial community of samples at the (A) phylum and (B) genus level. The bottom and top of the box are the first and third quartiles of the data, and the line inside the box is the median; the whiskers indicate the standard deviation; and the circles represent individual samples. Color version available online. 
Table 2. Bacterial composition of naturally fermented milks at the genus level

\begin{tabular}{lccrc}
\hline Genus & Cheese (\%) & Koumiss (\%) & Yak (\%) & Yogurt (\%) \\
\hline Lactobacillus & 1.12 & 82.75 & 78.44 & 64.69 \\
Lactococcus & 55.42 & 2.40 & 5.30 & 14.62 \\
Streptococcus & 31.90 & 13.93 & 14.08 & 10.29 \\
Acetobacter & 2.03 & 0.64 & 0.16 & 4.78 \\
Acinetobacter & 0.18 & 0.01 & 0.11 & 1.36 \\
Leuconostoc & 1.50 & 0.06 & 1.22 & 0.17 \\
Macrococcus & 0.71 & 0.02 & 0.05 & 0.38 \\
Enterococcus & 1.87 & 0.06 & 0.04 & 0.05 \\
Others & 5.28 & 0.12 & 0.61 & 3.67 \\
\hline
\end{tabular}

coccus detected in the cheese compared with the other sample types.

Besides sample type, our study looked at the influence of geographical origin in determining the microbial biodiversity of naturally fermented milk. We observed significant variations in the naturally fermented microbiota structure across sampling regions, which contradicts a previously published study that found no such difference in traditionally fermented milk products collected within Mongolia (Watanabe et al., 2008). One possible reason for the discrepancy may be the extent of the covered area. The geographical distance between sampling regions represents not only the physical distance between samples, but also environmental conditions such as temperature, humidity, and altitude. Other than sampling location and sample

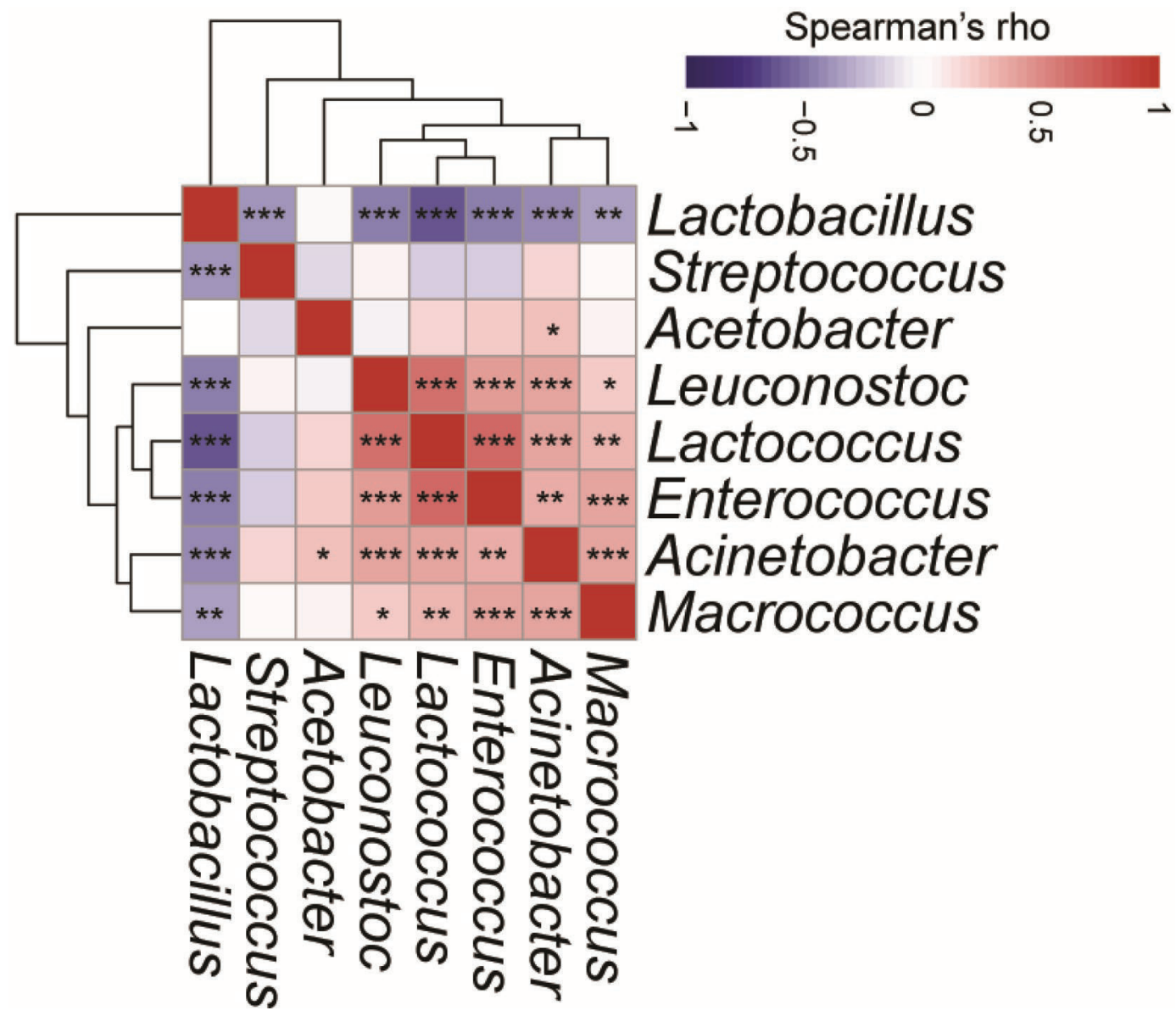

Figure 3. Correlation between some major genera identified in the naturally fermented milk. The Spearman rho is represented by the color scale, which ranges from a perfect positive (1) to a perfect negative correlation $(-1)$; ${ }^{* * *} P<0.001$, ${ }^{* *} P<0.01$, and ${ }^{*} P<0.05$, respectively. Color version available online. 

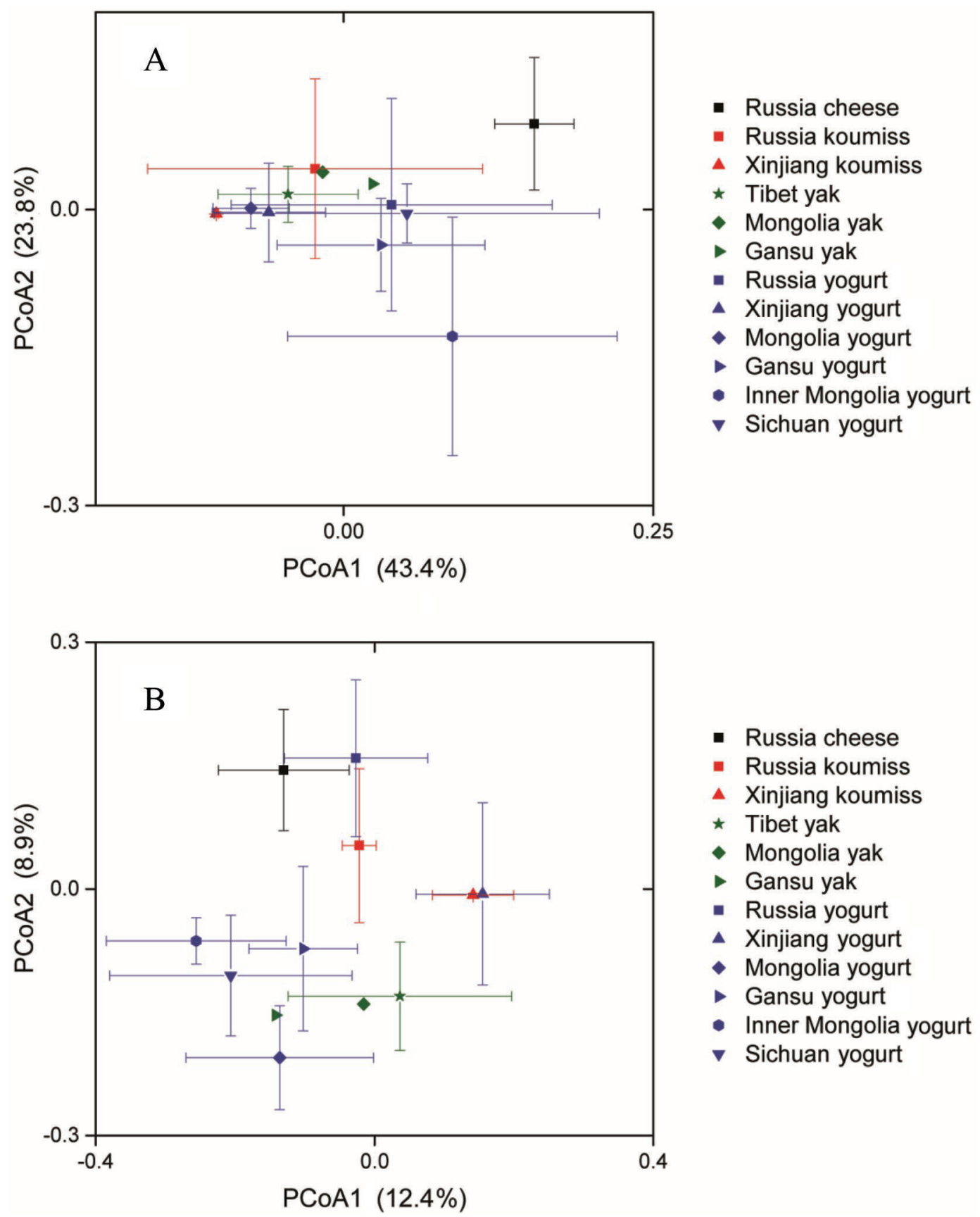

- Russia cheese

- Russia koumiss

- Xinjiang koumiss

* Tibet yak

- Mongolia yak

- Gansu yak

- Russia yogurt

- Xinjiang yogurt

- Mongolia yogurt

- Gansu yogurt

- Inner Mongolia yogurt

v Sichuan yogurt

Figure 4. Principal coordinate analysis (PCoA) plots of bacteria using (A) weighted and (B) unweighted UniFrac distances. Each point represents the mean principal component scores of samples at one location for one sample type; the horizontal and vertical error bars indicate the standard deviation of PCoA1 and PCoA2, respectively. Color version available online.

type, other circumstantial situations including the specific manufacturing steps, hygienic conditions, and inherited starter cultures may be involved in shaping the microbiota.

Apart from being one of the most prevalent populations within the fermented milk microbiota, Lacto- bacillus was negatively correlated with several genera, including Streptococcus, Lactococcus, Enterococcus, Acinetobacter, Leuconostoc, Acetobacter, and Macrococcus, suggesting that there may be some competition between Lactobacillus and other genera. It is a common industrial practice to use Lactobacillus and Streptococ- 

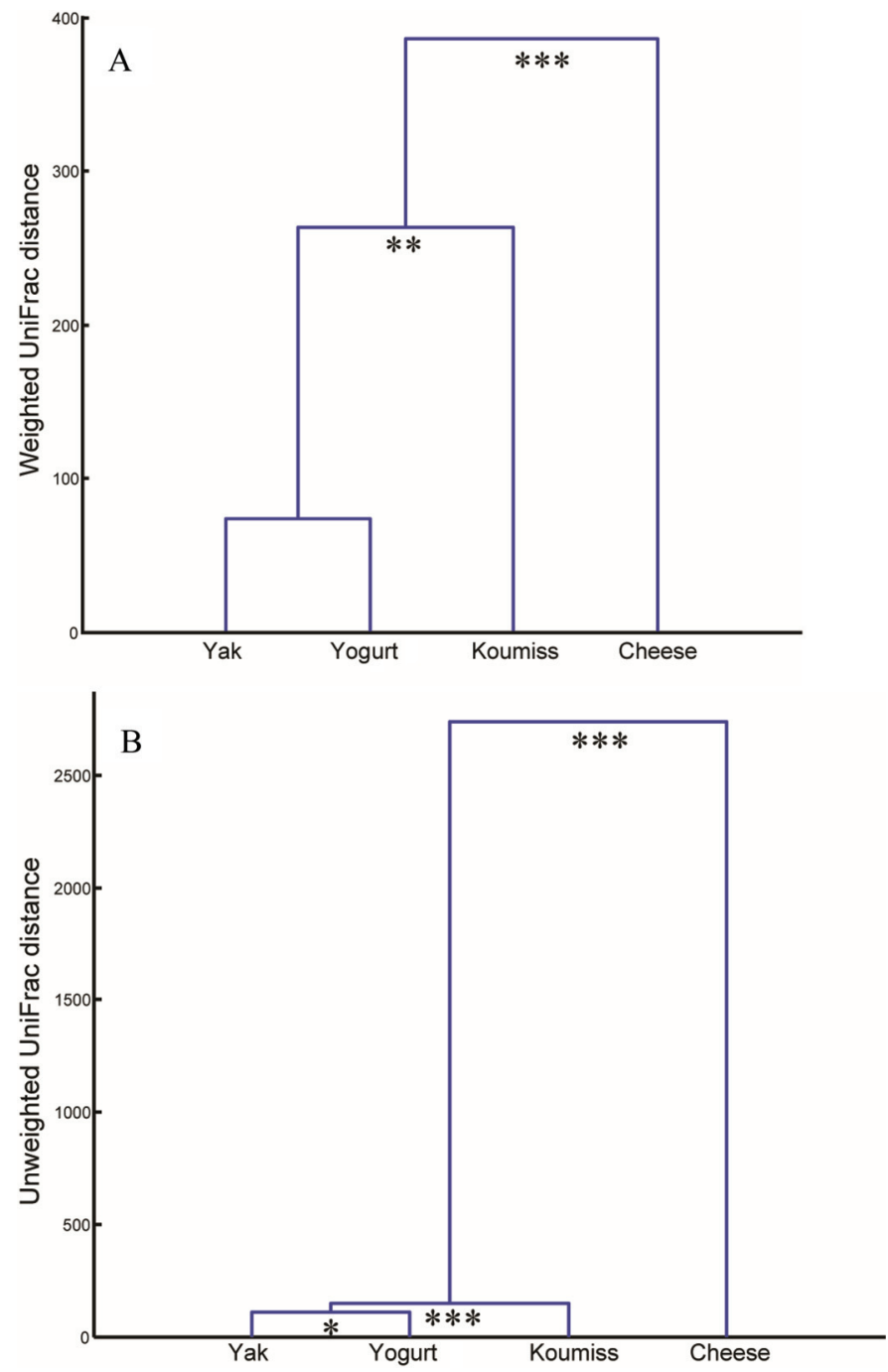

Figure 5. Clustering of different types of naturally fermented milk based on (A) weighted and (B) unweighted UniFrac distances calculated with multivariate ANOVA; ${ }^{* * *} P<0.001,{ }^{* *} P<0.01$, and ${ }^{*} P<$ 0.05 , respectively. Color version available online.

cus cocultures as the starters of fermented milk, as conventionally it has been thought that Lactobacillus and Streptococcus cooperate metabolically during the milk fermentation process. However, our results may indicate that these 2 genera are competitive and reach a dynamic equilibrium during the course of milk fermentation.

In conclusion, our study has provided an overview of the bacterial microbiota structure of naturally fermented milk based on pyrosequencing data of 85 samples collected over a wide geographic region, including China, Russia, and Mongolia. Our study collectively reanalyzed and consolidated the finding that both the type of naturally fermented milk and geographical origins are important in shaping the microbiota biodiversity of naturally fermented milk.

\section{ACKNOWLEDGMENTS}

This research was supported by the International S\&T Cooperation Program of China (Beijing; Grant No.2014DFR31150) and the China Agriculture Research System (Beijing; Grant No. CARS-37).

\section{REFERENCES}

Alegría, A., P. Szczesny, B. Mayo, J. Bardowski, and M. Kowalczyk. 2012. Biodiversity in Oscypek, a traditional Polish cheese, determined by culture-dependent and -independent approaches. Appl. Environ. Microbiol. 78:1890-1898.

Caporaso, J. G., J. Kuczynski, J. Stombaugh, K. Bittinger, F. D. Bushman, E. K. Costello, N. Fierer, A. G. Pena, J. K. Goodrich, J. I. Gordon, G. A. Huttley, S. T. Kelley, D. Knights, J. E. Koenig, R. E. Ley, C. A. Lozupone, D. McDonald, B. D. Muegge, M. Pirrung, J. Reeder, J. R. Sevinsky, P. J. Turnbaugh, W. A. Walters, J. Widmann, T. Yatsunenko, J. Zaneveld, and R. Knight. 2010 QIIME allows analysis of high-throughput community sequencing data. Nat. Methods 7:335-336

DeSantis, T. Z., P. Hugenholtz, N. Larsen, M. Rojas, E. L. Brodie, K. Keller, T. Huber, D. Dalevi, P. Hu, and G. L. Andersen. 2006. Greengenes, a chimera-checked $16 \mathrm{~S}$ rRNA gene database and workbench compatible with ARB. Appl. Environ. Microbiol. 72:5069-5072

Dobson, A., O. O'Sullivan, P. D. Cotter, P. Ross, and C. Hill. 2011. High-throughput sequence-based analysis of the bacterial composition of kefir and an associated kefir grain. FEMS Microbiol. Lett. 320:56-62.

Edgar, R. C. 2010. Search and clustering orders of magnitude faster than BLAST. Bioinformatics 26:2460-2461.

Ercolini, D., G. Moschetti, G. Blaiotta, and S. Coppola. 2001. The potential of a polyphasic PCR-dGGE approach in evaluating microbial diversity of natural whey cultures for water-buffalo Mozzarella cheese production: bias of culture-dependent and cultureindependent analyses. Syst. Appl. Microbiol. 24:610-617.

Giraffa, G., and E. Neviani. 2001. DNA-based, culture-independent strategies for evaluating microbial communities in food-associated ecosystems. Int. J. Food Microbiol. 67:19-34.

Haas, B. J., D. Gevers, A. M. Earl, M. Feldgarden, D. V. Ward, G. Giannoukos, D. Ciulla, D. Tabbaa, S. K. Highlander, E. Sodergren, B. Methe, T. Z. DeSantis, C. Human Microbiome, J. F. Petrosino, R. Knight, and B. W. Birren. 2011. Chimeric 16S rRNA sequence formation and detection in Sanger and 454-pyrosequenced PCR amplicons. Genome Res. 21:494-504.

Hols, P., F. Hancy, L. Fontaine, B. Grossiord, D. Prozzi, N. LeblondBourget, B. Decaris, A. Bolotin, C. Delorme, S. Dusko Ehrlich, E. Guedon, V. Monnet, P. Renault, and M. Kleerebezem. 2005. New insights in the molecular biology and physiology of Streptococcus thermophilus revealed by comparative genomics. FEMS Microbiol. Rev. 29:435-463.

Holzapfel, W. H. and B. J. B. Wood. 2014. Lactic Acid Bacteria: Biodiversity and Taxonomy. Wiley-Blackwell, Ames, IA.

Humblot, C., and J. P. Guyot. 2009. Pyrosequencing of tagged 16S rRNA gene amplicons for rapid deciphering of the microbiomes of fermented foods such as pearl millet slurries. Appl. Environ. Microbiol. 75:4354-4361.

Huys, G., M. Vancanneyt, K. D'Haene, V. Vankerckhoven, H. Goossens, and J. Swings. 2006. Accuracy of species identity of commercial bacterial cultures intended for probiotic or nutritional use. Res. Microbiol. 157:803-810. 
Jany, J. L., and G. Barbier. 2008. Culture-independent methods for identifying microbial communities in cheese. Food Microbiol. 25:839-848.

Kabuki, T., H. Uenishi, Y. Seto, T. Yoshioka, and H. Nakajima. 2009. A unique lantibiotic, thermophilin 1277, containing a disulfide bridge and two thioether bridges. J. Appl. Microbiol. 106:853-862.

Lattanzi, A., F. Minervini, R. Di Cagno, A. Diviccaro, L. Antonielli, G. Cardinali, S. Cappelle, M. De Angelis, and M. Gobbetti. 2013. The lactic acid bacteria and yeast microbiota of eighteen sourdoughs used for the manufacture of traditional Italian sweet leavened baked goods. Int. J. Food Microbiol. 163:71-79.

Leite, A. M., B. Mayo, C. T. Rachid, R. S. Peixoto, J. T. Silva, V. M. Paschoalin, and S. Delgado. 2012. Assessment of the microbial diversity of Brazilian kefir grains by PCR-DGGE and pyrosequencing analysis. Food Microbiol. 31:215-221.

Liu, W., Y. Zheng, L. Y. Kwok, Z. Sun, J. Zhang, Z. Guo, Q. Hou, B. Menhe, and H. Zhang. 2015a. High-throughput sequencing for the detection of the bacterial and fungal diversity in Mongolian naturally fermented cow's milk in Russia. BMC Microbiol. 15:45.

Liu, W. J., X. X. Xi, Q. G. Sudu, L. Y. Kwok, Z. Guo, Q. C. Hou, B. Menhe, T. S. Sun, and H. P. Zhang. 2015b. High-throughput sequencing reveals microbial community diversity of Tibetan naturally fermented yak milk. Ann. Microbiol. 65:1741-1751.

Lozupone, C., and R. Knight. 2005. UniFrac: A new phylogenetic method for comparing microbial communities. Appl. Environ. Microbiol. 71:8228-8235.

Marshall, V. M. E., and A. Y. Tamime. 1997. Physiology and biochemistry of fermented milks. Pages 153-192 in Microbiology and Biochemistry of Cheese and Fermented Milk. 2nd ed. B. A. Law, ed. Chapman \& Hall, London, UK.

Masoud, W., M. Takamiya, F. K. Vogensen, S. Lillevang, W. Abu Al-Soud, S. J. Sorensen, and M. Jakobsen. 2011. Characterization of bacterial populations in Danish raw milk cheeses made with different starter cultures by denaturating gradient gel electrophoresis and pyrosequencing. Int. Dairy J. 21:142-148.
Mayo, B., C. T. Rachid, A. Alegria, A. M. Leite, R. S. Peixoto, and S. Delgado. 2014. Impact of next generation sequencing techniques in food microbiology. Curr. Genomics 15:293-309.

Nam, Y. D., S. L. Park, and S. I. Lim. 2012. Microbial composition of the Korean traditional food "kochujang" analyzed by a massive sequencing technique. J. Food Sci. 77:M250-M256.

Oberman, H., and Z. Libudzisz. 1998. Fermented milks. Pages 308 350 in Microbiology of Fermented Foods. 2nd ed. B. J. Wood, ed. Blackie Academic \& Professional, London, UK.

Quigley, L., O. O'Sullivan, T. P. Beresford, R. P. Ross, G. F. Fitzgerald, and P. D. Cotter. 2012. High-throughput sequencing for detection of subpopulations of bacteria not previously associated with artisanal cheeses. Appl. Environ. Microbiol. 78:5717-5723.

Sakala, R. M., H. Hayashidani, Y. Kato, C. Kaneuchi, and M. Ogawa. 2002. Isolation and characterization of Lactococcus piscium strains from vacuum-packaged refrigerated beef. J. Appl. Microbiol. 92:173-179.

Sun, Z., W. Liu, Q. Bao, J. Zhang, Q. Hou, L. Kwok, T. Sun, and H. Zhang. 2014. Investigation of bacterial and fungal diversity in tarag using high-throughput sequencing. J. Dairy Sci. 97:60856096.

Watanabe, K., J. Fujimoto, M. Sasamoto, J. Dugersuren, T. Tumursuh, and S. Demberel. 2008. Diversity of lactic acid bacteria and yeasts in Airag and Tarag, traditional fermented milk products of Mongolia. World J Microb Biot. 24:1313-1325.

Xu, H., W. Liu, Q. Gesudu, Z. Sun, J. Zhang, Z. Guo, Y. Zheng, Q. Hou, J. Yu, Y. Qing, L. Y. Kwok, B. Menhe, and H. Zhang. 2015. Assessment of the bacterial and fungal diversity in home-made yoghurts of Xinjiang, China by pyrosequencing. J. Sci. Food Agric. 95:2007-2015.

Zamfir, M., M. Vancanneyt, L. Makras, F. Vaningelgem, K. Lefebvre, B. Pot, J. Swings, and L. De Vuyst. 2006. Biodiversity of lactic acid bacteria in Romanian dairy products. Syst. Appl. Microbiol. $29: 487-495$ 